UVX 2008 (2009) 107-111

(C) EDP Sciences, 2009

DOI: $10.1051 / \mathrm{uvx} / 2009017$

\title{
High-contrast pump-probe spectroscopy with high-order harmonics
}

\author{
Y. Mairesse ${ }^{1}$, W. Boutu ${ }^{2}$, P. Breger ${ }^{2}$, E. Constant ${ }^{1}$, D. Descamps ${ }^{1}$, \\ N. Dudovich ${ }^{3}$, B. Fabre ${ }^{1}$, S. Haessler ${ }^{2}$, J. Higuet ${ }^{1}$, E. Mével ${ }^{1}$, S. Petit ${ }^{1}$, \\ P. Salières ${ }^{2}$, D.M. Villeneuve ${ }^{4}$ and P.B. Corkum ${ }^{4}$ \\ ${ }^{1}$ CELIA, Université Bordeaux I, UMR 5107 (CNRS, Bordeaux 1, CEA), 351 cours de la \\ Libération, 33405 Talence Cedex, France \\ ${ }^{2}$ CEA-Saclay, DSM, Service des Photons, Atomes et Molécules, 91191 Gif-sur-Yvette, \\ France \\ ${ }^{3}$ Department of Physics of Complex Systems, Weizmann Institute of Science, Rehovot \\ 76100 , Israel \\ ${ }^{4}$ Steacie Institute for Molecular Sciences, National Research Council of Canada, 100 Sussex \\ Drive, Ottawa, Ontario K1A OR6, Canada
}

\begin{abstract}
The high order harmonic generation process from molecules can be used to probe the structure of the emitting medium with high spatial resolution $[1,2]$. The measurements of molecular dynamics with such a probe is thus very appealing. Contrast is an important issue in pump probe spectroscopy: in general the probe only excites part of the molecules, so that the detected signal can be dominated by contributions from non-excited molecules. In this article we review two techniques that can be used to enhance the contrast of pump probe measurements: transient grating spectroscopy and polarization-resolved spectroscopy. We use these techniques to detect rotational wavepackets and demonstrate a dramatic improvement in the contrast of the measured signal.
\end{abstract}

\section{INTRODUCTION}

High order harmonics can be produced by focusing an intense laser pulse into a gas jet. This process is not only a source of femtosecond and attosecond extreme ultraviolet (XUV) pulses; it also constitutes an accurate probe of the structure of the emitting medium. The harmonics are emitted when a strong-field driven electron wavepacket recollides to its parent ion [3,4]. During this recollision process, structural information on the molecule is encoded into the XUV spectrum. This information can be used to image molecular orbitals [1] or to measure molecular dynamics [2].

The sensitivity of high harmonic generation (HHG) to molecular dynamics was first demonstrated in the case of rotational wavepackets. When a femtosecond laser pulse is focused at about $10^{13} \mathrm{~W} / \mathrm{cm}^{2}$ in a molecular jet, it can coherently populate a superposition of rotational states - a rotational wavepacket - by Raman transitions [5,6]. As a result, the distribution of molecular alignment follows a particular evolution: every half rotational period of the considered species, the molecules are aligned along the laser polarization. The molecular dynamics can be probed by Coulomb explosion imaging [7] but also by high harmonic generation [8-10]: the harmonic signal shows modulations as the molecular alignment distribution evolves.

Using HHG as a probe of the emitting medium is quite similar to conventional nonlinear optical spectroscopy, in which a pump pulse excites a sample which is then probed by a second delayed pulse [11]. In such measurements, the pump pulse only excites a fraction of the molecules within the sample. The detected signal will thus in general contain an important continuous background from the non excited molecules. Enhancing the contrast in the detection is crucial to many pump-probe experiments. Several techniques have been developed in nonlinear optical spectroscopy. We have transposed two of them to the extremely nonlinear regime: transient grating spectroscopy [12] and polarization resolved 


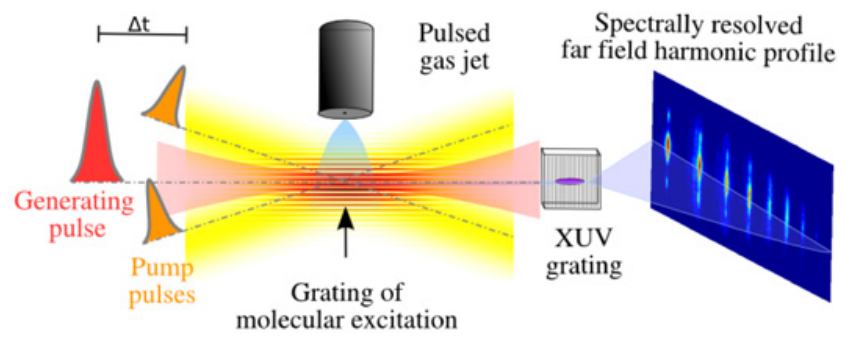

Figure 1. Experimental setup for high harmonic transient grating spectroscopy. Two crossed pump pulses create a grating of rotational excitation. A delayed probe pulse generates high harmonics in the excited medium. The emitted light is analyzed by an XUV spectrometer that images the spatial profile of each harmonic.

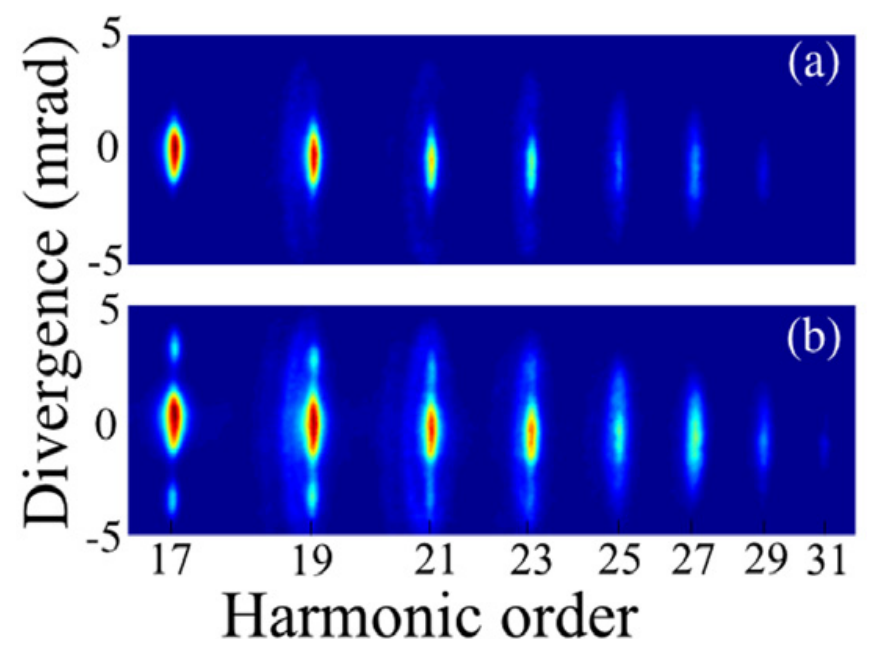

Figure 2. Spatially resolved harmonic spectrum generated in $\mathrm{N}_{2}$ at $1.5 \times 10^{14} \mathrm{~W} / \mathrm{cm}^{2}$, with isotropic molecular alignment (a) and at $\Delta t=4.1 \mathrm{ps}$ (b).

spectroscopy [13]. As a proof of principle, we consider the case of rotational wavepackets because their dynamics is well understood. However the extension of these techniques to other excitations is straightforward.

\section{TRANSIENT GRATING SPECTROSCOPY}

Transient grating spectroscopy (TGS) is one of the most commonly used techniques to perform background free nonlinear optical spectroscopy [11]. TGS is a four-wave mixing process in which the sample is excited by two synchronized non-colinear pump pulses. The resulting beam profile consists of interference fringes with a spacing dictated by the angle between the two pumps. Thus the excitation of the sample is spatially modulated: a grating of molecular excitation is created. The probe beam is focused on the sample and is diffracted by the grating. The detected signal is the diffracted light. The geometry is chosen such that there is no light emitted in the diffraction direction if there is no grating. Thus, the detected signal is zero when there is no excitation and the measurement is background free.

The experimental setup that was used to perform high harmonic TGS is shown in Fig. 1 [12]. The geometry is similar to conventional transient grating spectroscopy [14]. Two non-colinear pump pulses create a grating of rotational excitation in a pulsed molecular nitrogen gas jet. The fringe spacing is $13 \mu \mathrm{m}$. The rotational period of nitrogen molecules is $8.4 \mathrm{ps}$ and the first revival of molecular alignment 


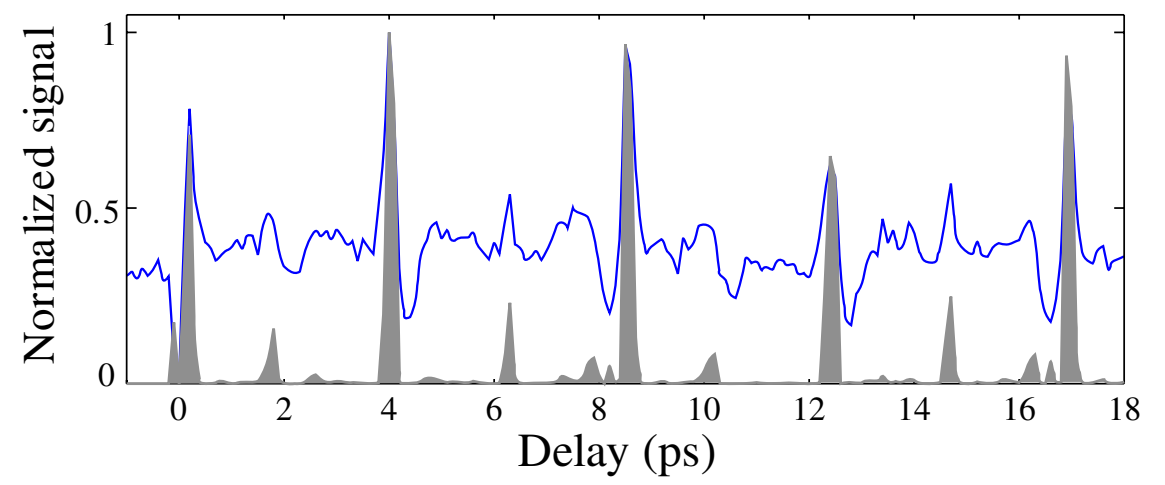

Figure 3. Evolution of the harmonic 17 total signal (line) and first order diffracted peak (gray shaded area) as a function of pump-probe delay.

is thus expected to occur around $4.2 \mathrm{ps}$. The central probe pulse is delayed by $\Delta t$ and focused on the grating with a waist at focus about $100 \mu \mathrm{m}$. It generates high harmonics which are analyzed by an XUV grating. The grating is cylindrical, so that it images the spectrum in the horizontal dimension without focusing the beam in the vertical dimension. The spectrally resolved spatial profile is imaged onto a set of MCPs associated to a phosphor screen and a CCD camera.

Figure 2(a) shows the spectrally resolved spatial profile obtained when the pump probe delay is negative, i.e. when there is no rotational excitation of the generating molecules. The harmonics consist of a central lobe and a more divergent part. They correspond respectively to short and long electron trajectories in the generation process. Figure 2(b) shows the picture obtained when the pump-probe delay is $\Delta t=4.1 \mathrm{ps}$. First order diffraction peaks appear on the sides of the lowest harmonics. The direction of these peaks is consistent with the fringe spacing of the excitation grating. The diffraction efficiency is strong for low orders (9.5\% of the energy in each diffraction peak for harmonic 17) and decreases as the harmonic order increases.

The evolution of the harmonic 17 signal as a function of pump probe delay is shown in Fig. 3 . The total signal follows the evolution of the degree of molecular alignment [7]: it increases when molecules are aligned and decreases when they are anti-aligned. The contrast in the detection of alignment revivals (ratio between signal from aligned and randomly aligned molecules) is less than a factor 3 . The grey shaded area shows the evolution of the diffracted light. The background in this signal is considerably reduced: the contrast in the detection of alignment is here more than 300:1. Such a high contrast enables us to detect subtle structures in the rotational wavepacket dynamics, which could not be seen in the total signal [12].

\section{POLARIZATION RESOLVED SPECTROSCOPY}

Polarization resolved spectroscopy is another technique that is commonly used to improve the contrast of pump-probe measurements. The principle of this technique, transposed to high harmonic generation, is shown in Fig. 4 [13]. When high harmonics are produced by an isotropic medium, the polarization of the harmonic electric field is parallel to that of the generating laser field. The orthogonal component $E_{\text {orth }}$ of the harmonic field is zero. As soon as there is an anisotropy in the generating medium, the polarization of the harmonics will in general be different from the laser polarization [15]. This leads to the appearence of a non-zero orthogonal component of the harmonic field. Thus, by detecting $E_{\text {orth }}$ rather than the total harmonic field, the contrast of the measurement can be increased.

The experimental setup for polarization-resolved pump probe spectroscopy is shown in Fig. 5. The laser beam is split in two in a delay line. The polarizations of the pump and probe beams can be adjusted 

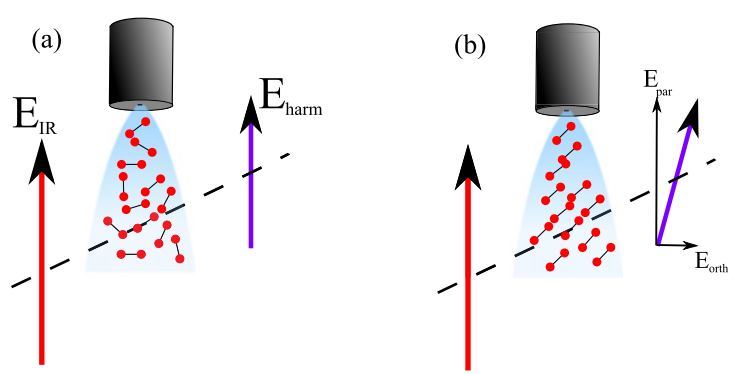

Figure 4. Principle of polarization resolved spectroscopy. In an isotropic medium, the harmonic polarization is parallel to the laser field (a). In an anisotropic medium this is in general not the case and an orthogonal electric field component can be produced (b).

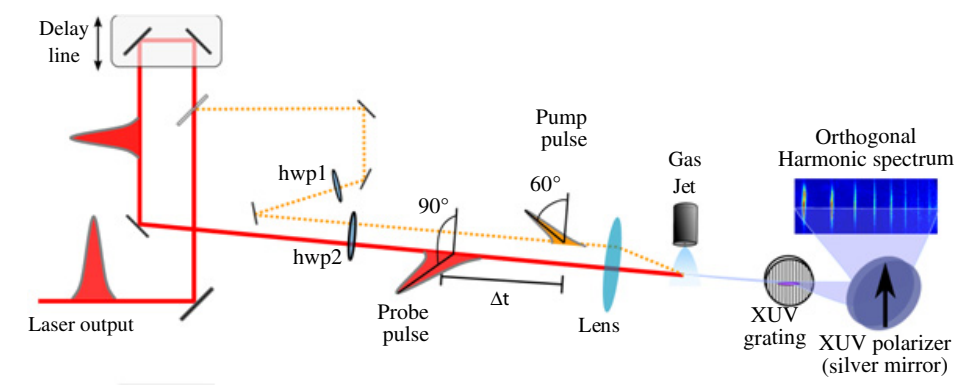

Figure 5. Experimental setup for polarization resolved pump probe spectroscopy. The probe beam polarization is orthogonal to the polarizer axis. The pump beam polarization is in between, so that symmetry is broken when molecules are aligned.

with two half waveplates. A silver mirror under $45^{\circ}$ incidence is placed between the grating and the detector. The reflectivity of this mirror is 30 times higher for $\mathrm{S}$ polarized incoming radiation than for $\mathrm{P}$ polarization. This mirror thus acts as an imperfect polarizer.

The polarization direction of the probe beam is chosen to be perpendicular to the axis of the polarizer. This configuration minimizes the detected signal when the medium is isotropic. The pump beam polarization is set to $60^{\circ}$ from the axis of the polarizer. This configuration is similar to the one presented in Fig. 4(b). We used a 50/50 mixture of argon and molecular nitrogen as a generating medium. Both gases have similar ionization potentials but the signal produced in pure argon is about 3 times stronger than the one from pure nitrogen. The signal from the mixture is thus dominated by contributions from argon.

The pump probe scan obtained with a standard configuration (i.e. no polarization resolution) is shown in Fig. 6 (gray line). It does not present significant modulations around the half-revival period of nitrogen. The signal obtained with polarization resolved spectroscopy is shown in black. A clear maximum is observed when molecules are aligned: polarization resolved spectroscopy enables us to extract an alignment signal from a strong background. The contrast of the maximum is 3.5 and is only limited by the extinction ratio of the polarizer. Using several silver mirrors would enable us to achieve a better contrast.

\section{CONCLUSION}

We have implemented two techniques to achieve high contrast pump probe spectroscopy with high harmonics: transient grating spectroscopy and polarization resolved spectroscopy. We have demonstrated a contrast enhancement by two orders of magnitude with TGS. Polarization resolved 


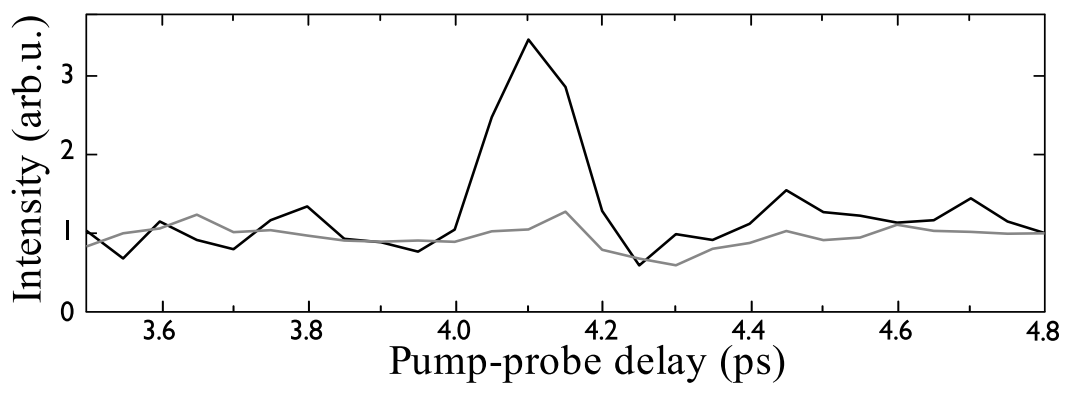

Figure 6. Pump-probe scan in a 50/50 mixture of nitrogen and argon. The black line was obtained with polarization resolved pump probe spectroscopy while the grey line was obtained with conventional detection.

spectroscopy has enabled us to extract the dynamics of rotational wavepackets in nitrogen from a strong continuous background generated by argon atoms.

In addition to this contrast enhancement, each technique presents additional advantages. The diffracted signal in TGS results from amplitude and phase modulations of the harmonic emission in the generating medium. The diffraction pattern can thus be used to extract the phase of the harmonic as a function of molecular alignment. The experimental setup used for polarization resolved spectroscopy can be used to measure the polarization state (direction, ellipticity) of the harmonics $[15,16]$. These parameters give precious information on the generating process and medium structure. While we have focused on the case of rotational wavepackets, it is clear that the two techniques implemented here will be useful to study other dynamics with high order harmonic generation.

\section{References}

[1] J. Itatani, J. Levesque, D. Zeidler, H. Niikura, H. Pépin, J. C. Kieffer, P. B. Corkum, and D. M. Villeneuve, Nature 432, 867 (2004).

[2] J.P. Marangos, S. Baker, N. Kajumba, J.S. Robinson, J.W.G Tisch, and R. Torres, Phys. Chem. Chem. Phys. 1035 (2008)

[3] P. B. Corkum, Phys. Rev. Lett. 71, 1994 (1993).

[4] K.J. Schafer, B. Yang, L.F. DiMauro and K.C. Kulander, Phys. Rev. Lett. 70, 1599 (1993).

[5] F. Rosca-Pruna and M. J. J. Vrakking, Phys. Rev. Lett. 87, 153902 (2001).

[6] H. Stapelfeldt and T. Seideman, Rev. Mod. Phys. 75, 543 (2003).

[7] P. W. Dooley, I. V. Litvinyuk, K. F. Lee, D. M. Rayner, M. Spanner, D. M. Villeneuve, and P. B. Corkum, Phys. Rev. A 68, 023406 (2003).

[8] T. Kanai, S. Minemoto and H. Sakai, Nature 435, 470 (2005).

[9] J. Itatani et al., Phys. Rev. Lett 94, 123902 (2005).

[10] C. Vozzi et al., Phys. Rev. Lett. 95, 153902 (2005).

[11] S. Mukamel, Principles of Nonlinear Optical Spectroscopy, Oxford University Press, New York, 1995

[12] Y. Mairesse , D. Zeidler, N. Dudovich, M. Spanner, J. Levesque, D.M. Villeneuve and P.B. Corkum, Phys. Rev. Lett. 100143903 (2008)

[13] Y. Mairesse, S. Haessler, B. Fabre, J. Higuet, W. Boutu, P. Breger, E. Constant, D. Descamps, E. Mével, S. Petit and P. Salières, New J. Phys. 10025028 (2008).

[14] A. Rouzée, V. Renard, S. Guérin, O. Faucher and B. Lavorel, Phys. Rev. A 75013419 (2007).

[15] J. Levesque, Y. Mairesse, N. Dudovich, H. Pépin, J.-C. Kieffer, P. B. Corkum, and D. M. Villeneuve, Phys. Rev. Lett 99, 243001 (2007).

[16] P. Antoine, B. Carré, A. L'Huillier, and M. Lewenstein, Phys. Rev. A 55, 1314 (1997). 